\title{
RESOURCE ALLOCATION FOR SUPPLY CHAINS BASED ON PARETO-OPTIMAL TWO-STAGE CROSS-EFFICIENCY MODEL
}

\author{
YUANYUAN ZHAO AND LEI FANG*
}

\begin{abstract}
The contradiction between the scarcity of common resources and the infinity of human demand for these resources has a significant impact on social development. Therefore, resource allocation can make the best use of limited resources in economic activities. Taking the two-stage supply chain where the outputs from the upstream supplier are taken as the inputs for the downstream manufacturer as an example, this paper applies the cross-efficiency model to comprehensively evaluate the efficiency scores of supply chains in the process of resource allocation and explores the relationship between the cross-efficiency of the supply chain and that of two enterprises within this supply chain. Furthermore, the self-interested behavior of enterprises is taken as the Pareto improvement principle to propose a Pareto-optimal two-stage cross-efficiency model, and this model can be used to optimally allocate the limited resources among two-stage supply chains. A common set of weights is determined to make all supply chains DEA efficient. Finally, the proposed model is illustrated to be feasible and effective through a practical application of 27 Iranian resin production companies.
\end{abstract}

Mathematics Subject Classification. 90B30.

Received October 11, 2021. Accepted February 21, 2022.

\section{INTRODUCTION}

In the increasingly competitive market environment, the supply chain has gradually become the mainstream trend of enterprise development. Specifically, different enterprises are combined into a functional chain through the strategy of complementary advantages to cope with the uncertainty of market demand and to obtain more economic benefits for these enterprises. The supply chain, which is composed of multiple enterprises cooperating with each other, can not only reduce unnecessary costs in the process of providing products or services but also promote enterprises to improve their rapid response to changes in market demand through information sharing, so as to maximize the benefits of the entire supply chain and to promote the efficient development of the social economy. However, it is obvious that the scarcity of resources is becoming increasingly serious with the development of human society, both in terms of natural and social resources. Therefore, the optimal allocation of limited resources can not only maximize the utility of resources but also appropriately alleviate the contradiction between the scarcity of common resources and the infinity of human demand for these resources in social development, which can promote sustainable development of the world. Wu et al. [27]

Keywords. Data envelopment analysis, two-stage cross-efficiency model, Pareto optimality, resource allocation.

Department of Management Science and Engineering, Business school, Nankai university, No. 121 Baidi Road, Nankai District, Tianjin300071, P.R. China.

*Corresponding author: fanglei@nankai.edu.cn 
studied a resource allocation strategy in the supply chain of manufacturing resources by proposing a matrixbased Bayesian approach and employing a genetic algorithm to determine an optimal and robust manufacturing resource allocation scheme within the context specified by the user. In addition, Zhu et al. [33], with the support of big data technology, proposed the SBM-DEA model to evaluate the utilization efficiency of natural resources for 26 provincial regions in mainland China from 2005 to 2012, and a DEA-based approach was developed to allocate a reduced total of natural resources among the 26 provincial regions. Sharahi and Khalili-Damghani [21] developed a decision support system based on the concepts of the benchmark in DEA and the optimal design in De-Novo programming, and the optimal allocation of resources and optimal values of the targets are set in Iranian natural gas supply chains under a real-world scenario where the budget of resources and targets are usually mixed with uncertainties. An et al. [1] proposed a fixed cost allocation approach for basic two-stage systems based on the principle of efficiency invariance, and investigated the related allocation plans for twostage systems under cooperative and noncooperative scenarios. Li et al. [14] addressed the fixed cost allocation problem among a set of DMUs considering undesired outputs based on the joint weak disposability assumption, and developed a satisfaction degree bargaining game approach to determine the existence of a unique allocation scheme such that all DMUs can be simultaneously efficient. Furthermore, Zhao et al. [32] distinguished four patterns of resource allocation and applied simulation from evolutionary game theory to reveal the stability of various resource allocations under different constraint situations, so as to explore the effects of different resource allocations in the development of strategic alliances.

Whether resource allocation is reasonable or not has an obvious impact on the level of socioeconomic development. Efficiency is one of the criteria to measure the development level of organization systems. Data envelopment analysis (DEA), which was originally introduced by [3], is driven by objective data to evaluate the relative efficiency of a group of homogeneous decision making units (DMUs) with multiple inputs and multiple outputs. This non-parametric method can not only determine the structure of the envelopment frontier without artificially defining parameters in advance but also effectively avoid the unscientific weight schemes caused by artificial assignment. However, the traditional DEA models measure the self-evaluated efficiency of DMUs. That is, these DMUs choose a set of optimal input-output weights to maximize their own efficiency scores based on the self-interested principle. As a result, there are multiple efficient DMUs with an efficiency score of 1 , which does not facilitate the ranking among efficient DMUs.

To improve the power of traditional DEA models in discriminating efficient DMUs, Sexton et al. [22] introduced the concept of peer evaluation into traditional DEA models and proposed the cross-efficiency model to measure the cross-efficiency scores of DMUs. This comprehensive evaluation method analyzes not only the selfevaluated efficiency of DMUs, but also the peer-evaluated efficiency obtained by using the optimal input-output weights of other DMUs. Since there are multiple optimal solutions for the input-output weights generated from the traditional DEA models, the cross-efficiency results of DMUs calculated by the optimal input-output weights are generally not unique. To avoid this problem, Doyle and Green [9] introduced some secondary objectives to develop the benevolent (or aggressive) cross-efficiency model, which can maximize (or minimize) the aggregate efficiency of all DMUs except the evaluated DMU. Furthermore, Liang et al. [16] considered that each DMU seeks to maximize its own efficiency under the condition that the cross-efficiency scores of other DMUs do not deteriorate, and proposed a DEA game cross-efficiency model to obtain the optimal game cross-efficiency scores, which are proved to constitute a Nash equilibrium point. Wang and Chin [25] proposed a neutral cross-efficiency model to determine a set of input-output weights for each DMU from its own viewpoint, without involving benevolent or aggressive attitudes toward the other DMUs, and this model can effectively reduce the number of zero weights for the output indicators. On this basis, Wang et al. [26] proposed an extended neutral cross-efficiency model, which can reduce the number of zero weights in terms of both input and output indicators. In addition, Lim [17] proposed the new aggressive (or benevolent) cross-efficiency model to solve the non-uniqueness of optimal weights by taking the minimization (or maximization) of the cross-efficiency of the best (or worst) peer DMUs as the secondary goal. Wu et al. [29] incorporated a target identification model to determine the reachable targets for all DMUs, and proposed several secondary goal models to select a set of optimal input-output weights by considering the DMUs willingness to get close to their desirable cross-efficiency targets and to avoid their 
undesirable cross-efficiency targets. However, Cook and Zhu [7] pointed out that meaningful secondary goal models can develop nonlinear programming problems, which can increase the computational difficulty of these models, and thus a unit-invariant multiplicative DEA model is proposed to calculate the cross-efficiency scores of DMUs, so as to eliminate the need for imposing secondary goals to solve the non-uniqueness problem of optimal weights. Moreover, Chen et al. [5] introduced meta-frontier technology into the cross-efficiency evaluation model to explore the technology gap between different frontiers in the cross-efficiency environment and to solve the overestimation problem of the self-evaluation method, and then chose the aggressive cross-evaluation strategy to solve the non-uniqueness of optimal solutions. In addition, Xie et al. [30] proposed Shannons entropy DEA model to provide a complete ranking for all DMUs, and the important input and output variables are selected by using the Bayesian information criterion and the ordinary least squares regression technology.

The above extended cross-efficiency models only measure the production efficiency of the single-stage structure system and do not take into account the internal structure of that system. However, in the real-world environment, as the supply chain mentioned above, it is an operational process in which multiple enterprises cooperate with each other to provide products or services. As a result, the efficiency score of the supply chain measured by these evaluation models that ignores the internal structure of the supply chain is not reasonable, and there may be a phenomenon in which the supply chain is efficient but the enterprises within this supply chain are inefficient. Therefore, Örkcü et al. [20] extended the research of [26] to basic two-stage network systems and proposed a new neutral cross-efficiency model that can not only effectively reduce the number of zero weights for inputs, intermediate products and outputs, but also have improved discrimination power to fully rank the DMUs in both overall and substages of two-stage network systems. In addition, Kao and Liu [11] applied the concept of cross evaluation to measure the cross-efficiency scores of two basic network systems, and the results show that the cross-efficiency of a system can be decomposed into the product of those of the divisions for the series structure and a weighted average of those of the divisions for the parallel structure. Moreover, Ma et al. [18] revealed that the centralized model proposed by [15] from the perspective of cooperative game theory may lead to multiple and unacceptable cross-efficiency scores, and developed a game cross-efficiency model based on the non-cooperative game theory to obtain a unique cross-efficiency result for the overall systems and two subsystems. Moreover, Shao and Wang [23] introduced prospect theory into the two-stage cross-efficiency evaluation method. In addition, Meng and Xiong [19] applied the concept of multiplicative hesitant fuzzy preference relations (MHFPRs) and acceptable consistency analysis to calculate the priority of DMUs, and the overall efficiency of the general two-stage system can be decomposed from the self-evaluation and peer-evaluation perspective.

However, there is still another important issue in the cross-efficiency evaluation; that is, the generated crossefficiency scores are not Pareto optimal, which makes it difficult for the cross-efficiency evaluation results to be accepted by all DMUs. In the process of cross-efficiency evaluation, the so-called Pareto-optimal means that there is no situation in which the cross-efficiency score of any DMU is increased without allowing the cross-efficiency score of at least one DMU to decrease. In other words, the improvement of the cross-efficiency score of a DMU is achieved at the cost of decreasing the cross-efficiency of at least one DMU other than it. In this case, all DMUs cannot further improve their cross-efficiency scores by Pareto improvement. Furthermore, Wu et al. [28] proposed the Pareto optimality estimation model to determine whether the given set of crossefficiency scores are Pareto-optimal solutions, and the Pareto improvement model is developed to make the cross-efficiency score of the evaluated DMU better off without making any DMUs cross-efficiency scores worse off. Furthermore, Davtalab-Olyaie and Asgharian [8] proposed a multi-objective programming model based on the self-prioritizing principle, and applied the weighted sum technique to develop a linear model, which can determine an optimal weights profile to calculate the Pareto-optimal cross-efficiency scores of all DMUs. And [24] used the cross-efficiency method based on a secondary goal model to allocate fixed costs, and it is also verified that the performance results of all DMUs are Pareto cross-efficient after the allocation.

To eliminate unrealistic weighting schemes provided by DEA models with a self-evaluated method in the process of resource allocation and to avoid unreasonable performance results of DMUs after the resource allocation, extended cross-efficiency models have also been widely used to explore the optimal allocation of common resources. Du et al. [10] used the cross-efficiency concept in DEA to approach cost and resource allocation 
problems, and the cross-efficiency iterative method was proposed to maximize the aggregated output change by allocating available resources. Subsequently, Yu et al. [31] combined a two-stage network DEA model and the concept of cross-efficiency to solve the fixed cost allocation problem in the two-stage process, and the iterative method of [10] was applied to determine the optimal cross-efficiency scores for two stages and to obtain the optimal fixed cost allocation among all DMUs. Furthermore, Li et al. [13] proposed a DEA game cross-efficiency approach to address the fixed cost allocation problem by explicitly considering both competition and cooperation relationships among DMUs, and the Shapley value was used to generate a unique and fair allocation scheme. Moreover, Chen et al. [6] introduced the preference for all individual DMUs into proposing a new centralized resource allocation strategy based on the cross-efficiency iterative method. In addition, Davtalab-Olyaie and Asgharian [8] applied the Pareto-optimal cross-efficiency model to the R\&D project selection, and the total budget was allocated among all projects based on the Pareto-optimal results, which can improve resource allocation and fund more projects. In the existing research, however, there are few studies on the development of the Pareto-optimal cross-efficiency model from the perspective of enterprises self-interested principle to explore the optimal allocation strategy of common resources among two-stage structure systems, and the relationship between the cross-efficiency of the overall system and that of subsystems in the resource allocation environment also needs to be further studied.

Therefore, taking the basic two-stage supply chain as the research object, this paper introduces the advantages of the cross-evaluated method and the concept of Pareto-optimality into the DEA-based method, and proposes the Pareto-optimal two-stage cross-efficiency model to achieve the resource allocation among two-stage supply chains by taking the self-interested motivation of DMUs who prefer to maximize their own interests as the Pareto improvement principle. Then, the relationship between the cross-efficiency of the overall system and that of subsystems is analyzed, and the cross-efficiency results of supply chains after the implementation of the resource allocation strategy are explored. Finally, the feasibility and effectiveness of the proposed model are illustrated by using an application example for coordinating sustainable supply chains. The contributions of this paper are mainly reflected in three aspects. First of all, the optimal results of resource allocation obtained from the perspective of maximizing enterprises interests are more easily accepted by the two-stage supply chains. Secondly, in the resource allocation environment, the cross-efficiency of the system can be decomposed into the weighted sum of those of subsystems for the two-stage supply chain. And thirdly, the proposed model can not only achieve the optimal allocation of limited resources, but also make the Pareto-optimal cross-efficiency of all two-stage supply chains achieve DEA efficient after the allocation.

In addition, actual supply chains always take the most favorable decision behavior to maximize their own interests. Then in the resource allocation process, all supply chains also want to obtain the optimal amount of common resources and to determine the optimal input-output weights so as to maximize their own operational efficiencies. However, the resource allocation strategy determined by the above self-evaluated approach based on the self-interested principle is difficult to be accepted by all supply chains other than itself. Therefore, the current paper introduces the peer-evaluated concept to carry out cross-evaluation among supply chains. And in the cross-evaluation process that takes into account the self-interested behavior of supply chains, it is possible to guarantee that the cross-efficiency scores of supply chains will not become worse by at least making the self-evaluated efficiency scores of each supply chain not lower than its peer-evaluated efficiency scores generated from the other supply chains. As a result, this paper takes the above self-interested behavior as the Pareto improvement conditions and allows all supply chains to choose the same weights, so that all supply chains can not only determine an optimal allocation strategy of common resources but also obtain Pareto-optimal crossefficiency scores under this strategy. This approach is consistent with the self-interested motivation of supply chains, which can facilitate the implementation of resource allocation strategy.

The remainder of this paper is organized as follows. Section 2 applies a cross-efficiency model to measure the cross-efficiency for the overall system and two enterprises within that system in the process of resource allocation, and then explores the relationship between the cross-efficiency of the supply chain and that of two enterprises within this supply chain. Section 3 proposes the two-stage cross-efficiency model based on Pareto-optimality in the resource allocation environment. Section 4 explores the optimal allocation strategy of common resources 
among the sustainable supply chains of 27 Iranian resin production companies, and determines the Paretooptimal cross-efficiency results of supply chains after the allocation. Finally, Section 5 offers the conclusion and research prospect.

\section{Cross-efficiency of Supply Chains under Resource Allocation}

In this section, we approach the allocation scheme of common resources among two-stage supply chains based on the self-evaluated and peer-evaluated methods. Suppose that there are $n$ homogeneous two-stage supply chains, where the outputs from the upstream supplier are called intermediate products and they are also taken as the inputs for the downstream manufacturer having no other additional inputs. It should be noted that the two-stage supply chains studied in the paper can be called DMUs in the research scope of DEA. In supply chain $\mathrm{DMU}_{j}(j=1,2, \ldots, n)$, the upstream supplier consumes $m$ initial inputs $X_{j}=\left(x_{1 j}, x_{2 j}, \ldots, x_{m j}\right)^{T} \in R_{+}^{m}$ to produce $D$ intermediate products $Z_{j}=\left(z_{1 j}, z_{2 j}, \ldots, z_{D j}\right)^{T} \in R_{+}^{D}$, and the downstream manufacturer uses these $D$ intermediate products to generate $s$ ultimate outputs $Y_{j}=\left(y_{1 j}, y_{2 j}, \ldots, y_{s j}\right)^{T} \in R_{+}^{s}$. In addition, there are common resources $\hat{R}$, which need to be appropriately allocated among $n$ two-stage supply chains, and these common resources are materials, funds, human resources and information resources required for the operation of supply chains. Furthermore, the amount of common resources allocated to $\operatorname{DMU}_{j}(j=1,2, \ldots, n)$ is $\hat{R}_{j}(j=1,2, \ldots, n)$. Where, the amount of resources allocated to the upstream supplier is $\hat{R}_{1 j}$, and the amount of resources allocated to the downstream manufacturer is $\hat{R}_{2 j}$. As a result, the operation process of the two-stage supply chain in the resource allocation environment is shown in Figure 1.

This paper applies the multiplier form of the DEA model to determine a set of optimal weights with the objective of maximizing the operational performance of the entire supply chain, thereby identifying the amount of resources allocated to each supply chain and measuring the efficiency scores of two-stage supply chains after the resource allocation. In this paper, since the system efficiency of a two-stage supply chain is the weighted sum of the stage efficiency of the upstream supplier and that of the downstream manufacturer, the system efficiency of the supply chain can be measured by model (2.1). And noted that we define $\operatorname{DMU}_{k}(k=1,2, \ldots, n)$ as an evaluated supply chain.

$$
\begin{aligned}
& \max \quad E_{k k}^{(\mathrm{S})}=\omega_{k k}^{(1)} \cdot E_{k k}^{(1)}+\omega_{k k}^{(2)} \cdot E_{k k}^{(2)} \\
& \text { s.t. } \quad E_{k j}^{(1)}=\frac{\Phi_{k}^{T} Z_{j}}{V_{k}^{T} X_{j}+W_{k} \cdot \hat{R}_{1 j}} \leq 1, j=1,2, \ldots, n \text {; } \\
& E_{k j}^{(2)}=\frac{U_{k}^{T} Y_{j}}{\Phi_{k}^{T} Z_{j}+W_{k} \cdot \hat{R}_{2 j}} \leq 1, j=1,2, \ldots, n ; \\
& \sum_{j=1}^{n}\left(\hat{R}_{1 j}+\hat{R}_{2 j}\right)=\hat{R}
\end{aligned}
$$

$$
D M U_{j}(j=1,2, \ldots, m)
$$

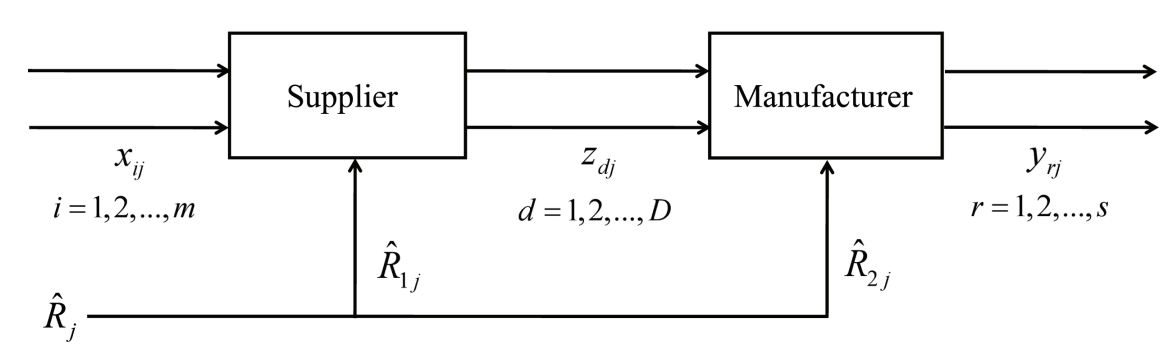

FIGURE 1. The basic two-stage supply chain in the process of resource allocation. 


$$
\begin{gathered}
\hat{R}_{1 j} \geq 0, \hat{R}_{2 j} \geq 0, j=1,2, \ldots, n \\
V_{k} \geq \mathbf{0}, U_{k} \geq \mathbf{0}, \Phi_{k} \geq \mathbf{0}, W_{k}>0, k=1,2, \ldots, n
\end{gathered}
$$

Where $E_{k k}^{(1)}$ and $E_{k k}^{(2)}$ represent the efficiency of the upstream supplier and that of the downstream manufacturer after the resource allocation, respectively. Furthermore, $V_{k}=\left(v_{1 k}, v_{2 k}, \ldots, v_{m k}\right)^{T} \in R_{+}^{m}$ represents the weight vector of the initial inputs for the upstream supplier, $U_{k}=\left(u_{1 k}, u_{2 k}, \ldots, u_{s k}\right)^{T} \in R_{+}^{s}$ represents the weight vector of the ultimate outputs for the downstream manufacturer, $\Phi_{k}=\left(\varphi_{1 k}, \varphi_{2 k}, \ldots, \varphi_{D k}\right)^{T} \in R_{+}^{D}$ is the weight vector of the intermediate products, and $\mathbf{0}$ is a zero vector. Here, this paper sets the same weight vector of intermediate products for both enterprises, which can avoid the potential conflict about intermediate products within the two-stage supply chain. Furthermore, since the type of a common resource is the same for both the upstream supplier and the downstream manufacturer, the indicator weight of common resources is denoted by $W_{k}$ for both the upstream supplier and the downstream manufacturer.

In the objective function of model $(2.1), \omega_{k k}^{(1)}$ and $\omega_{k k}^{(2)}$ correspond to the stage weight of the upstream supplier and that of the downstream manufacturer in the evaluated supply chain $\mathrm{DMU}_{k}$. With reference to the weight expression proposed by [4], the ratio of the weighted inputs of an enterprise to those of the whole supply chain is applied as the stage weight of this enterprise, and the specific expression is shown below.

$$
\begin{aligned}
\omega_{k k}^{(1)} & =\frac{V_{k}^{T} X_{k}+W_{k} \cdot \hat{R}_{1 k}}{V_{k}^{T} X_{k}+W_{k} \cdot \hat{R}_{1 k}+\Phi_{k}^{T} Z_{k}+W_{k} \cdot \hat{R}_{2 k}} ; \\
\omega_{k k}^{(2)} & =\frac{\Phi_{k}^{T} Z_{k}+W_{k} \cdot \hat{R}_{2 k}}{V_{k}^{T} X_{k}+W_{k} \cdot \hat{R}_{1 k}+\Phi_{k}^{T} Z_{k}+W_{k} \cdot \hat{R}_{2 k}}
\end{aligned}
$$

Based on this, expression (2.2) is introduced into the objective function of model (2.1), and then model (2.1) can be further transformed into the following fractional programming model (2.3).

$$
\begin{array}{ll}
\max & E_{k k}^{(\mathrm{S})}=\frac{\Phi_{k}^{T} Z_{k}+U_{k}^{T} Y_{k}}{V_{k}^{T} X_{k}+W_{k} \cdot \hat{R}_{1 k}+\Phi_{k}^{T} Z_{k}+W_{k} \cdot \hat{R}_{2 k}} \\
\text { s.t. } & \frac{\Phi_{k}^{T} Z_{j}+U_{k}^{T} Y_{j}}{V_{k}^{T} X_{j}+W_{k} \cdot \hat{R}_{1 j}+\Phi_{k}^{T} Z_{j}+W_{k} \cdot \hat{R}_{2 j}} \leq 1, j=1,2, \ldots, n ; \\
& \frac{\Phi_{k}^{T} Z_{j}}{V_{k}^{T} X_{j}+W_{k} \cdot \hat{R}_{1 j}} \leq 1, j=1,2, \ldots, n ; \\
& \frac{U_{k}^{T} Y_{j}}{\Phi_{k}^{T} Z_{j}+W_{k} \cdot \hat{R}_{2 j}} \leq 1, j=1,2, \ldots, n ; \\
& \sum_{j=1}^{n}\left(\hat{R}_{1 j}+\hat{R}_{2 j}\right)=\hat{R} ; \\
& \hat{R}_{1 j} \geq 0, \hat{R}_{2 j} \geq 0, j=1,2, \ldots, n ; \\
& V_{k} \geq \mathbf{0}, U_{k} \geq \mathbf{0}, \Phi_{k} \geq \mathbf{0}, W_{k}>0, k=1,2, \ldots, n ;
\end{array}
$$

By solving model (2.3), the optimal weights profile $\left(V_{k}^{* T}, U_{k}^{* T}, \Phi_{k}^{* T}, W_{k}^{*}\right)$ for $\operatorname{DMU}_{k}(k=1,2, \ldots, n)$ can be obtained. Then, the cross-efficiency of $\mathrm{DMU}_{j}$ relative to $\mathrm{DMU}_{k}$ in terms of the whole system and two stages can be calculated as in expression (2.4). 


$$
\begin{aligned}
E_{k j}^{(\mathrm{S}) *} & =\frac{\Phi_{k}^{* T} Z_{j}+U_{k}^{* T} Y_{j}}{V_{k}^{* T} X_{j}+W_{k}^{*} \cdot \hat{R}_{1 j}+\Phi_{k}^{* T} Z_{j}+W_{k}^{*} \cdot \hat{R}_{2 j}} ; \\
E_{k j}^{(1) *} & =\frac{\Phi_{k}^{* T} Z_{j}}{V_{k}^{* T} X_{j}+W_{k}^{*} \cdot \hat{R}_{1 j}} ; \\
E_{k j}^{(2) *} & =\frac{U_{k}^{T} Y_{j}}{\Phi_{k}^{T} Z_{j}+W_{k} \cdot \hat{R}_{2 j}} .
\end{aligned}
$$

Subsequently, the relationship between the cross-efficiency of the overall system and that of two enterprises within that system is further explored. Similar to the objective function of the self-evaluated model (2.1), the peer-evaluated efficiency $E_{k j}^{(\mathrm{S})}$ of the supply chain can be further decomposed as a weighted sum of the peerevaluated efficiency $E_{k j}^{(1)}$ of the upstream supplier and the peer-evaluated efficiency $E_{k j}^{(2)}$ of the downstream manufacturer, as follows:

$$
\begin{aligned}
E_{k j}^{(\mathrm{S})} & =\frac{\Phi_{k}^{T} Z_{j}+U_{k}^{T} Y_{j}}{V_{k}^{T} X_{j}+W_{k} \cdot \hat{R}_{1 j}+\Phi_{k}^{T} Z_{j}+W_{k} \cdot \hat{R}_{2 j}} \\
& =\frac{V_{k}^{T} X_{j}+W_{k} \cdot \hat{R}_{1 j}}{V_{k}^{T} X_{j}+W_{k} \cdot \hat{R}_{1 j}+\Phi_{k}^{T} Z_{j}+W_{k} \cdot \hat{R}_{2 j}} \times \frac{\Phi_{k}^{T} Z_{j}}{V_{k}^{T} X_{j}+W_{k} \cdot \hat{R}_{1 j}} \\
& +\frac{\Phi_{k}^{T} Z_{j}+W_{k} \cdot \hat{R}_{2 j}}{V_{k}^{T} X_{j}+W_{k} \cdot \hat{R}_{1 j}+\Phi_{k}^{T} Z_{j}+W_{k} \cdot \hat{R}_{2 j}} \times \frac{U_{k}^{T} Y_{j}}{\Phi_{k}^{T} Z_{j}+W_{k} \cdot \hat{R}_{2 j}} \\
& =\omega_{k j}^{(1)} \cdot E_{k j}^{(1)}+\omega_{k j}^{(2)} \cdot E_{k j}^{(2)}
\end{aligned}
$$

In model (2.5), $\omega_{k j}^{(1)}$ represents the peer-evaluated stage weight of $\mathrm{DMU}_{j}$ relative to $\mathrm{DMU}_{k}$ in terms of the upstream supplier, and $\omega_{k j}^{(2)}$ represents the peer-evaluated stage weight of $\mathrm{DMU}_{j}$ relative to $\mathrm{DMU}_{k}$ in terms of the downstream manufacturer.

The above processes determine self-efficiency and peer-efficiency for both supply chains and two enterprises within the supply chain. And then, the cross-efficiency scores of supply chains and those of enterprises are further measured. Specifically, this paper identifies the cross-efficiency results for two enterprises prior to solving for the cross-efficiency scores of supply chains. Therefore, the cross-efficiency $\tilde{E}_{j}^{(1)}$ of the upstream supplier is determined by the weighted sum of the self-evaluated efficiency $E_{j j}^{(1)}$ and the peer-evaluated efficiency $E_{k j}^{(1)}(k=1,2, \ldots, n ; k \neq j)$, and the weight is denoted as $\beta_{k j}^{(1)}=\omega_{k j}^{(1)} / \sum_{k=1}^{n} \omega_{k j}^{(1)}$, which satisfies $\sum_{k=1}^{n} \beta_{k j}^{(1)}=1$ and $\beta_{k j}^{(1)} \geq 0(k=1,2, \ldots, n)$. As a result, the cross-efficiency $\tilde{E}_{j}^{(1)}$ of the upstream supplier is expressed as:

$$
\tilde{E}_{j}^{(1)}=\sum_{k=1}^{n} \beta_{k j}^{(1)} \cdot E_{k j}^{(1)}=\sum_{k=1}^{n}\left(\frac{\omega_{k j}^{(1)}}{\sum_{k=1}^{n} \omega_{k j}^{(1)}} \cdot E_{k j}^{(1)}\right)=\frac{\sum_{k=1}^{n} \omega_{k j}^{(1)} \cdot E_{k j}^{(1)}}{\sum_{k=1}^{n} \omega_{k j}^{(1)}}
$$

Similarly, the cross-efficiency $\tilde{E}_{j}^{(2)}$ of the downstream manufacturer can be expressed as:

$$
\tilde{E}_{j}^{(2)}=\sum_{k=1}^{n} \beta_{k j}^{(2)} \cdot E_{k j}^{(2)}=\sum_{k=1}^{n}\left(\frac{\omega_{k j}^{(2)}}{\sum_{k=1}^{n} \omega_{k j}^{(2)}} \cdot E_{k j}^{(2)}\right)=\frac{\sum_{k=1}^{n} \omega_{k j}^{(2)} \cdot E_{k j}^{(2)}}{\sum_{k=1}^{n} \omega_{k j}^{(2)}}
$$


Next, we determine the cross-efficiency $E_{j}^{(\mathrm{S})}$ of the supply chain $\mathrm{DMU}_{j}$. Specifically, the cross-efficiency $E_{j}^{(\mathrm{S})}$ of supply chain is the arithmetic average of the self-evaluated efficiency $E_{j j}^{(\mathrm{S})}$ and the peer-evaluated efficiency $E_{k j}^{(\mathrm{S})}$, that is, $\bar{E}_{j}^{(\mathrm{S})}=\frac{1}{n} \sum_{k=1}^{n} E_{k j}^{(\mathrm{S})}$.

From the objective function of model (2.1), we can determine that the self-evaluated efficiency of the overall system can be decomposed into the weighted sum of the self-evaluated efficiency of two enterprises for the two-stage supply chain. And as expressed in expression (2.5), this weighted sum technique is also applicable in the peer-evaluated method. Therefore, this paper sets the cross-evaluated stage weight of the upstream supplier and that of the downstream manufacturer as $\bar{\omega}_{j}^{(1)}=\frac{1}{n} \sum_{k=1}^{n} \omega_{k j}^{(1)}$ and $\bar{\omega}_{j}^{(2)}=\frac{1}{n} \sum_{k=1}^{n} \omega_{k j}^{(2)}$ respectively, so that the cross-efficiency of the supply chain also satisfies this weighted sum technique; that is, the cross-efficiency of the supply chain is the weighted sum of the cross-efficiency of the upstream supplier and that of the downstream manufacturer. The details are shown as follows.

$$
\begin{aligned}
& \bar{\omega}_{j}^{(1)} \cdot \tilde{E}_{j}^{(1)}+\bar{\omega}_{j}^{(2)} \cdot \tilde{E}_{j}^{(2)}=\frac{1}{n} \sum_{k=1}^{n} \omega_{k j}^{(1)} \cdot \frac{\sum_{k=1}^{n} \omega_{k j}^{(1)} \cdot E_{k j}^{(1)}}{\sum_{k=1}^{n} \omega_{k j}^{(1)}}+\frac{1}{n} \sum_{k=1}^{n} \omega_{k j}^{(2)} \cdot \frac{\sum_{k=1}^{n} \omega_{k j}^{(2)} \cdot E_{k j}^{(2)}}{\sum_{k=1}^{n} \omega_{k j}^{(2)}} \\
& =\frac{1}{n} \cdot \sum_{k=1}^{n} \omega_{k j}^{(1)} \cdot E_{k j}^{(1)}+\frac{1}{n} \cdot \sum_{k=1}^{n} \omega_{k j}^{(2)} \cdot E_{k j}^{(2)}=\frac{1}{n} \cdot \sum_{k=1}^{n}\left(\omega_{k j}^{(1)} \cdot E_{k j}^{(1)}+\omega_{k j}^{(2)} \cdot E_{k j}^{(2)}\right)=\frac{1}{n} \cdot \sum_{k=1}^{n} E_{k j}^{(\mathrm{S})}=\bar{E}_{j}^{(\mathrm{S})}
\end{aligned}
$$

As a result, the cross-efficiency of one enterprise can be decomposed into the weighted sum of its selfevaluated efficiency and its peer-evaluated efficiency. And the cross-efficiency of a two-stage supply chain can be decomposed into the weighted sum of the cross-efficiency of the upstream supplier and the cross-efficiency of the downstream manufacturer, where the stage weight of each enterprise is the cross-weight obtained by the arithmetic average of the self-evaluated weight and the peer-evaluated weight.

\section{Two-stage CROSS-EFficiency MOdel BASEd on PARETO-OPtimality in the RESOURCE ALLOCATION ENVIRONMENT}

In this section, we develop a new two-stage cross-efficiency model with Pareto-optimality to address the problem of resource allocation among two-stage supply chains. The proposed model not only determines a set of optimal weights to obtain Pareto-optimal cross-efficiency scores for all supply chains, but also provides an optimal strategy of resource allocation to make all supply chains DEA efficient after the allocation.

Each supply chain and the two enterprises in the supply chain aim to maximize their own interests in the resource allocation process. And since the upstream supplier and the downstream manufacturer are important components of a two-stage supply chain studied in this paper, decision makers in both enterprises make the decisions that are most beneficial to them in the process of efficiency evaluation. Therefore, it is possible to ensure that the cross-efficiency scores of the supply chain do not deteriorate by satisfying the situation where at least the self-evaluated efficiency of this supply chain is not lower than its peer-evaluated efficiency. The same is true for the upstream supplier and the downstream manufacturer. As a result, the following three Paretooptimal conditions need to be satisfied in the process of determining the optimal weights vector of the supply chain.

$$
\begin{aligned}
& E_{k j}^{(\mathrm{S})} \leq E_{j j}^{(\mathrm{S})}, \forall k, j \quad \& \quad k \neq j ; \\
& E_{k j}^{(1)} \leq E_{j j}^{(1)}, \forall k, j \quad \& \quad k \neq j ; \\
& E_{k j}^{(2)} \leq E_{j j}^{(2)}, \forall k, j \quad \& \quad k \neq j ;
\end{aligned}
$$


Therefore, based on the above Pareto-optimal principle, a multi-objective programming model (3.2) is developed to calculate the Pareto-optimal cross-efficiency scores of all supply chains, and the optimal allocation of common resources can also be determined.

$$
\begin{array}{ll}
\max & \left\{\bar{E}_{1}^{(\mathrm{S})}, \bar{E}_{2}^{(\mathrm{S})}, \ldots, \bar{E}_{n}^{(\mathrm{S})}\right\} \\
\text { s.t. } & E_{k j}^{(\mathrm{S})}=\frac{\Phi_{k}^{T} Z_{j}+U_{k}^{T} Y_{j}}{V_{k}^{T} X_{j}+W_{k} \cdot \hat{R}_{1 j}+\Phi_{k}^{T} Z_{j}+W_{k} \cdot \hat{R}_{2 j}} \\
& \leq E_{j j}^{(\mathrm{S})}=\frac{\Phi_{j}^{T} Z_{j}+U_{j}^{T} Y_{j}}{V_{j}^{T} X_{j}+W_{j} \cdot \hat{R}_{1 j}+\Phi_{j}^{T} Z_{j}+W_{j} \cdot \hat{R}_{2 j}}, \forall k, j \quad \& \quad k \neq j ; \\
& E_{k j}^{(1)}=\frac{\Phi_{k}^{T} Z_{j}}{V_{k}^{T} X_{j}+W_{k} \cdot \hat{R}_{1 j}} \leq E_{j j}^{(1)}=\frac{\Phi_{j}^{T} Z_{j}}{V_{j}^{T} X_{j}+W_{j} \cdot \hat{R}_{1 j}}, \forall k, j \quad \& \quad k \neq j ; \\
& E_{k j}^{(2)}=\frac{U_{k}^{T} Y_{j}}{\Phi_{k}^{T} Z_{j}+W_{k} \cdot \hat{R}_{2 j}} \leq E_{j j}^{(2)}=\frac{U_{j}^{T} Y_{j}}{\Phi_{j}^{T} Z_{j}+W_{j} \cdot \hat{R}_{2 j}}, \forall k, j \quad \& \quad k \neq j ; \\
& \sum_{j=1}^{n}\left(\hat{R}_{1 j}+\hat{R}_{2 j}\right)=\hat{R} ; \\
& \hat{R}_{1 j} \geq 0, \hat{R}_{2 j} \geq 0, j=1,2, \ldots, n ; \\
& \bar{E}_{j}^{(\mathrm{S})}=\frac{1}{n} \sum_{k=1}^{n} E_{k j}^{(\mathrm{S})}, j=1,2, \ldots, n ; \\
& E_{k j}^{(\mathrm{S})}=\frac{\Phi_{k}^{T} Z_{j}+U_{k}^{T} Y_{j}}{V_{k}^{T} X_{j}+W_{k} \cdot \hat{R}_{1 j}+\Phi_{k}^{T} Z_{j}+W_{k} \cdot \hat{R}_{2 j}} \leq 1, k=1,2, \ldots, n, j=1,2, \ldots, n ; \\
& E_{k j}^{(1)}=\frac{\Phi_{k}^{T} Z_{j}}{V_{k}^{T} X_{j}+W_{k} \cdot \hat{R}_{1 j}} \leq 1, k=1,2, \ldots, n, j=1,2, \ldots, n ; \\
& E_{k j}^{(2)}=\frac{U_{k}^{T} Y_{j}}{\Phi_{k}^{T} Z_{j}+W_{k} \cdot \hat{R}_{2 j}} \leq 1, k=1,2, \ldots, n, j=1,2, \ldots, n ; \\
& V_{k} \geq \mathbf{0}, U_{k} \geq \mathbf{0}, \Phi_{k} \geq \mathbf{0}, W_{k}>0, k=1,2, \ldots, n ;
\end{array}
$$

The purpose of model (3.2) is to maximize the cross-efficiency scores of all supply chains. Therein, the first three constraints about Pareto-optimality ensure that the self-evaluated efficiency scores are not less than the peer-evaluated efficiency scores for supply chains and two enterprises within the supply chain. The fourth and fifth constraints ensure that the total amount of common resources is $\hat{R}$. And in the sixth constraint, $\bar{E}_{j}^{(\mathrm{S})}$ denotes the cross-efficiency score of the supply chain $\mathrm{DMU}_{j}$. In addition, the seventh to ninth constraints require that the peer-evaluated efficiency score of the supply chain and the peer-evaluated efficiency scores of two enterprises within this supply chain are not greater than 1, which ensures that the peer-evaluated process is consistent with the realistic situation. As a result, the Pareto-optimal weights profile $\left(V_{k}^{\prime T},{U_{k}^{\prime}}^{T}, \Phi_{k}^{\prime T}, W^{\prime}{ }_{k}, k=1,2, \ldots, n\right)$ can be measured, and thus the Pareto-optimal cross-efficiency scores $\bar{E}_{j}^{(\mathrm{S})^{\prime}}$ can be obtained.

To solve this multi-objective programming model, this paper takes the preferred degree $\alpha_{j}$ of the supply chain $\mathrm{DMU}_{j}$ into account, and coverts the model (3.2) into a single-objective programming model by applying the weighted sum technique. 


$$
\begin{array}{ll}
\max & \sum_{j=1}^{n} \sum_{k=1}^{n} \alpha_{j} \cdot \frac{1}{n} E_{k j}^{(\mathrm{S})} \\
\text { s.t. } & E_{k j}^{(\mathrm{S})} \leq E_{j j}^{(\mathrm{S})}, k=1,2, \ldots, n, j=1,2, \ldots, n, k \neq j ; \\
& E_{k j}^{(1)} \leq E_{j j}^{(1)}, k=1,2, \ldots, n, j=1,2, \ldots, n, k \neq j ; \\
& E_{k j}^{(2)} \leq E_{j j}^{(2)}, k=1,2, \ldots, n, j=1,2, \ldots, n, k \neq j ; \\
& \sum_{j=1}^{n}\left(\hat{R}_{1 j}+\hat{R}_{2 j}\right)=\hat{R} ; \\
& \hat{R}_{1 j} \geq 0, \hat{R}_{2 j} \geq 0, j=1,2, \ldots, n ; \\
& E_{k j}^{(\mathrm{S})} \leq 1, k=1,2, \ldots, n, j=1,2, \ldots, n ; \\
& E_{k j}^{(1)} \leq 1, k=1,2, \ldots, n, j=1,2, \ldots, n ; \\
& E_{k j}^{(2)} \leq 1, k=1,2, \ldots, n, j=1,2, \ldots, n ; \\
& V_{k} \geq \mathbf{0}, U_{k} \geq \mathbf{0}, \Phi_{k} \geq \mathbf{0}, W_{k}>0, k=1,2, \ldots, n ;
\end{array}
$$

However, model (3.3) is still a nonlinear programming model, and this model needs to be further transformed into a linear programming model that is convenient to calculate. Therefore, there are two theorems about model (3.3) that need to be illustrated in advance.

Theorem 3.1. In model (3.3), all supply chains choose a Pareto-optimal weights vector, which makes all supply chains obtain the Pareto-optimal cross-efficiency scores.

Proof. Assume that $\left(\bar{V}_{k}^{T}, \bar{U}_{k}^{T}, \bar{\Phi}_{k}^{T}, \bar{W}_{k}, k=1,2, \ldots, n\right)$ is an optimal solution of model (3.3), and we have

$$
\sum_{k=1}^{n} \frac{\bar{U}_{l}^{T} Y_{k}+\bar{\Phi}_{l}^{T} Z_{k}}{\bar{V}_{l}^{T} X_{k}+\bar{W}_{l} \cdot \hat{R}_{1 k}+\bar{\Phi}_{l}^{T} Z_{k}+\bar{W}_{l} \cdot \hat{R}_{2 k}}=\max _{j}\left\{\sum_{k=1}^{n} \frac{\bar{U}_{j}^{T} Y_{k}+\bar{\Phi}_{j}^{T} Z_{k}}{\bar{V}_{j}^{T} X_{k}+\bar{W}_{j} \cdot \hat{R}_{1 k}+\bar{\Phi}_{j}^{T} Z_{k}+\bar{W}_{j} \cdot \hat{R}_{2 k}}\right\} .
$$

Let $\left(\hat{V}_{k}^{T}, \hat{U}_{k}^{T}, \hat{\Phi}_{k}^{T}, \hat{W}_{k}\right)=\left(\bar{V}_{l}^{T}, \bar{U}_{l}^{T}, \bar{\Phi}_{l}^{T}, \bar{W}_{l}\right)$ for all supply chain $D M U_{k}(k=1,2, \ldots, n)$. Since we have

$$
\begin{aligned}
& \frac{\hat{U}_{k}^{T} Y_{j}+\hat{\Phi}_{k}^{T} Z_{j}}{\hat{V}_{k}^{T} X_{j}+\hat{W}_{k} \cdot \hat{R}_{1 j}+\hat{\Phi}_{k}^{T} Z_{j}+\hat{W}_{k} \cdot \hat{R}_{2 j}}=\frac{\bar{U}_{l}^{T} Y_{j}+\bar{\Phi}_{l}^{T} Z_{j}}{\bar{V}_{l}^{T} X_{j}+\bar{W}_{l} \cdot \hat{R}_{1 j}+\bar{\Phi}_{l}^{T} Z_{j}+\bar{W}_{l} \cdot \hat{R}_{2 j}} \\
& \leq \frac{\hat{U}_{j}^{T} Y_{j}+\hat{\Phi}_{j}^{T} Z_{j}}{\hat{V}_{j}^{T} X_{j}+\hat{W}_{j} \cdot \hat{R}_{1 j}+\hat{\Phi}_{j}^{T} Z_{j}+\hat{W}_{j} \cdot \hat{R}_{2 j}}=\frac{\bar{U}_{l}^{T} Y_{j}+\bar{\Phi}_{l}^{T} Z_{j}}{\bar{V}_{l}^{T} X_{j}+\bar{W}_{l} \cdot \hat{R}_{1 j}+\bar{\Phi}_{l}^{T} Z_{j}+\bar{W}_{l} \cdot \hat{R}_{2 j}}
\end{aligned}, \forall k, j,
$$

the first constraint in model (3.3) also holds for $\left(\hat{V}_{k}^{T}, \hat{U}_{k}^{T}, \hat{\Phi}_{k}^{T}, \hat{W}_{k}, k=1,2, \ldots, n\right)$. And it also satisfies the second and third constraints of model (3.3), since we have $\frac{\hat{\Phi}_{k}^{T} Z_{j}}{\hat{V}_{k}^{T} X_{j}+\hat{W}_{k} \cdot \hat{R}_{1 j}}=\frac{\bar{\Phi}_{l}^{T} Z_{j}}{\bar{V}_{l}^{T} X_{j}+\bar{W}_{l} \cdot \hat{R}_{1 j}} \leq \frac{\hat{\Phi}_{j}^{T} Z_{j}}{\hat{V}_{j}^{T} X_{j}+\hat{W}_{j} \cdot \hat{R}_{1 j}}=$ $\frac{\bar{\Phi}_{l}^{T} Z_{j}}{\bar{V}_{l}^{T} X_{j}+\bar{W}_{l} \cdot \hat{R}_{1 j}}, \forall k, j$ and $\frac{\hat{U}_{k}^{T} Y_{j}}{\hat{\Phi}_{k}^{T} Z_{j}+\hat{W}_{k} \cdot \hat{R}_{2 j}}=\frac{\bar{U}_{l}^{T} Y_{j}}{\bar{\Phi}_{l}^{T} Z_{j}+\bar{W}_{l} \cdot \hat{R}_{2 j}} \leq \frac{\hat{U}_{j}^{T} Y_{j}}{\hat{\Phi}_{j}^{T} Z_{j}+\hat{W}_{j} \cdot \hat{R}_{2 j}}=\frac{\bar{U}_{l}^{T} Y_{j}}{\bar{\Phi}_{l}^{T} Z_{j}+\bar{W}_{l} \cdot \hat{R}_{2 j}}, \forall k, j$.

Furthermore, $\left(\hat{V}_{k}^{T}, \hat{U}_{k}^{T}, \hat{\Phi}_{k}^{T}, \hat{W}_{k}, k=1,2, \ldots, n\right)$ also satisfies the fifth to seventh constraints of model (3.3), as reflected in the following three expressions.

$$
\begin{aligned}
& \hat{U}_{k}^{T} Y_{j}+\hat{\Phi}_{k}^{T} Z_{j}-\hat{V}_{k}^{T} X_{j}-\hat{W}_{k} \cdot \hat{R}_{1 j}-\hat{\Phi}_{k}^{T} Z_{j}-\hat{W}_{k} \cdot \hat{R}_{2 j} \\
& =\bar{U}_{l}^{T} Y_{j}+\bar{\Phi}_{l}^{T} Z_{j}-\bar{V}_{l}^{T} X_{j}-\bar{W}_{l} \cdot \hat{R}_{1 j}-\bar{\Phi}_{l}^{T} Z_{j}-\bar{W}_{l} \cdot \hat{R}_{2 j} \leq 0, \forall k, j ;
\end{aligned}
$$




$$
\begin{aligned}
& \hat{\Phi}_{k}^{T} Z_{j}-\hat{V}_{k}^{T} X_{j}-\hat{W}_{k} \cdot \hat{R}_{1 j}=\bar{\Phi}_{l}^{T} Z_{j}-\bar{V}_{l}^{T} X_{j}-\bar{W}_{l} \cdot \hat{R}_{1 j} \leq 0, \forall k, j ; \\
& \hat{U}_{k}^{T} Y_{j}-\hat{\Phi}_{k}^{T} Z_{j}-\hat{W}_{k} \cdot \hat{R}_{2 j}=\bar{U}_{l}^{T} Y_{j}-\bar{\Phi}_{l}^{T} Z_{j}-\bar{W}_{l} \cdot \hat{R}_{2 j} \leq 0, \forall k, j
\end{aligned}
$$

Therefore, $\left(\hat{V}_{k}^{T}, \hat{U}_{k}^{T}, \hat{\Phi}_{k}^{T}, \hat{W}_{k}, k=1,2, \ldots, n\right)$ is a feasible solution of model (3.3). Then, the objective function of model (3.3) based on this feasible solution can be expressed as

$$
\begin{aligned}
& \sum_{j=1}^{n} \sum_{k=1}^{n} \alpha_{j} \cdot \frac{1}{n} \cdot \frac{\hat{U}_{k}^{T} Y_{j}+\hat{\Phi}_{k}^{T} Z_{j}}{\hat{V}_{k}^{T} X_{j}+\hat{W}_{k} \cdot \hat{R}_{1 j}+\hat{\Phi}_{k}^{T} Z_{j}+\hat{W}_{k} \cdot \hat{R}_{2 j}}=n \sum_{j=1}^{n} \alpha_{j} \cdot \frac{1}{n} \cdot \frac{\bar{U}_{l}^{T} Y_{j}+\bar{\Phi}_{l}^{T} Z_{j}}{\bar{V}_{l}^{T} X_{j}+\bar{W}_{l} \cdot \hat{R}_{1 j}+\bar{\Phi}_{l}^{T} Z_{j}+\bar{W}_{l} \cdot \hat{R}_{2 j}} \\
& \geq \sum_{j=1}^{n} \sum_{k=1}^{n} \alpha_{j} \cdot \frac{1}{n} \cdot \frac{\bar{U}_{k}^{T} Y_{j}+\bar{\Phi}_{k}^{T} Z_{j}}{\bar{V}_{k}^{T} X_{j}+\bar{W}_{k} \cdot \hat{R}_{1 j}+\bar{\Phi}_{k}^{T} Z_{j}+\bar{W}_{k} \cdot \hat{R}_{2 j}}
\end{aligned}
$$

As a result, $\left(\hat{V}_{k}^{T}, \hat{U}_{k}^{T}, \hat{\Phi}_{k}^{T}, \hat{W}_{k}, k=1,2, \ldots, n\right)$ is also an optimal solution of model (3.3). Hence, there is a common set of weights in model (3.3) that makes all supply chains obtain Pareto-optimal cross-efficiency scores.

On the basis of Theorem 3.1, this paper marks the common weights profile determined by model (3.3) as $\left(V^{T}, U^{T}, \Phi^{T}, W\right)$. Furthermore, model (3.3) can be further adjusted to be model (3.4) under the common weights profile.

$$
\begin{array}{ll}
\max & \sum_{j=1}^{n} \alpha_{j} \cdot \frac{U^{T} Y_{j}+\Phi^{T} Z_{j}}{V^{T} X_{j}+W \cdot \hat{R}_{1 j}+\Phi^{T} Z_{j}+W \cdot \hat{R}_{2 j}} \\
\text { s.t. } & \frac{U^{T} Y_{j}+\Phi^{T} Z_{j}}{V^{T} X_{j}+W \cdot \hat{R}_{1 j}+\Phi^{T} Z_{j}+W \cdot \hat{R}_{2 j}} \leq 1, j=1,2, \ldots, n ; \\
& \frac{\Phi^{T} Z_{j}}{V^{T} X_{j}+W \cdot \hat{R}_{1 j}} \leq 1, j=1,2, \ldots, n \\
& \frac{U^{T} Y_{j}}{\Phi^{T} Z_{j}+W \cdot \hat{R}_{2 j}} \leq 1, j=1,2, \ldots, n ; \\
& \sum_{j=1}^{n}\left(\hat{R}_{1 j}+\hat{R}_{2 j}\right)=\hat{R} ; \hat{R}_{1 j} \geq 0, \hat{R}_{2 j} \geq 0, j=1,2, \ldots, n ; \\
& V \geq \mathbf{0}, U \geq \mathbf{0}, \Phi \geq \mathbf{0}, W>0 .
\end{array}
$$

Since there is a common set of weights in model (3.4), $\omega_{k j}^{(1)}=\omega_{j j}^{(1)}=\omega^{(1)}$ and $\omega_{k j}^{(2)}=\omega_{j j}^{(2)}=\omega^{(2)}$ hold. And then, $\omega^{(1)} \cdot E_{k j}^{(1)}+\omega^{(2)} \cdot E_{k j}^{(2)} \leq \omega^{(1)} \cdot E_{j j}^{(1)}+\omega^{(2)} \cdot E_{j j}^{(2)}$ is also satisfied in the peer evaluation for the supply chain. Therefore, $E_{k j}^{(\mathrm{S})} \leq E_{j j}^{(\mathrm{S})}$ also holds when the upstream supplier and the downstream manufacturer satisfy $E_{k j}^{(1)} \leq E_{j j}^{(1)}$ and $E_{k j}^{(2)} \leq E_{j j}^{(2)}$, respectively.

It should be emphasized that model (3.4) remains a nonlinear programming model even with the common weights profile. Therefore, based on the weight expression proposed by [8], this paper reflects the preferred degree of DMU $j$ relative to all supply chains by defining the portion of the total resources devoted to DMU $j$. Specifically, let $\sum_{d=1}^{n}\left(V^{T} X_{d}+W \cdot \hat{R}_{1 d}+\Phi^{T} Z_{d}+W \cdot \hat{R}_{2 d}\right)$ as the total size consumed by all two-stage supply chains, and let $V^{T} X_{j}+W \cdot \hat{R}_{1 j}+\Phi^{T} Z_{j}+W \cdot \hat{R}_{2 j}$ as the size of DMU $j$. Then the current paper defines $\alpha_{j}$ as $\frac{V^{T} X_{j}+W \cdot \hat{R}_{1 j}+\Phi^{T} Z_{j}+W \cdot \hat{R}_{2 j}}{\sum_{d=1}^{n}\left(V^{T} X_{d}+W \cdot \hat{R}_{1 d}+\Phi^{T} Z_{d}+W \cdot \hat{R}_{2 d}\right)}$, which represents the proportion of the weighted inputs of DMU $j$ among those 
of all DMUs. Therefore, the objective function of model (3.4) can be converted as shown below.

$$
\begin{aligned}
& \sum_{j=1}^{n} \alpha_{j} \cdot \frac{U^{T} Y_{j}+\Phi^{T} Z_{j}}{V^{T} X_{j}+W \cdot \hat{R}_{1 j}+\Phi^{T} Z_{j}+W \cdot \hat{R}_{2 j}}=\frac{\sum_{j=1}^{n}\left(U^{T} Y_{j}+\Phi^{T} Z_{j}\right)}{\sum_{d=1}^{n}\left(V^{T} X_{d}+W \cdot \hat{R}_{1 d}+\Phi^{T} Z_{d}+W \cdot \hat{R}_{2 d}\right)} \\
& =\frac{U^{T} \cdot\left(\sum_{j=1}^{n} Y_{j}\right)+\Phi^{T} \cdot\left(\sum_{j=1}^{n} Z_{j}\right)}{V^{T} \cdot\left(\sum_{j=1}^{n} X_{j}\right)+\Phi^{T} \cdot\left(\sum_{j=1}^{n} Z_{j}\right)+W \cdot \hat{R}}
\end{aligned}
$$

Moreover, we convert the above model (3.4) into model (3.5) by using the Charnes-Cooper transformation (2). Model (3.5) can obtain an optimal weights profile for all supply chains to generate a set of the Pareto-optimal cross-efficiency scores and to determine the optimal strategy of resource allocation.

$$
\begin{array}{ll}
\max & U^{T} \cdot\left(\sum_{j=1}^{n} Y_{j}\right)+\Phi^{T} \cdot\left(\sum_{j=1}^{n} Z_{j}\right) \\
\text { s.t. } & V^{T} \cdot\left(\sum_{j=1}^{n} X_{j}\right)+\Phi^{T} \cdot\left(\sum_{j=1}^{n} Z_{j}\right)+W \cdot \hat{R}=1 ; \\
& U^{T} Y_{j}-V^{T} X_{j}-W \cdot \hat{R}_{1 j}-W \cdot \hat{R}_{2 j} \leq 0, j=1,2, \ldots, n ; \\
& \Phi^{T} Z_{j}-V^{T} X_{j}-W \cdot \hat{R}_{1 j} \leq 0, j=1,2, \ldots, n ; \\
& U^{T} Y_{j}-\Phi^{T} Z_{j}-W \cdot \hat{R}_{2 j} \leq 0, j=1,2, \ldots, n ; \\
& \sum_{j=1}^{n}\left(\hat{R}_{1 j}+\hat{R}_{2 j}\right)=\hat{R} ; \\
& \hat{R}_{1 j} \geq 0, \hat{R}_{2 j} \geq 0, j=1,2, \ldots, n ; \\
& V \geq \mathbf{0}, U \geq \mathbf{0}, \Phi \geq \mathbf{0}, W>0 .
\end{array}
$$

Since $W \cdot \hat{R}_{1 j}$ and $W \cdot \hat{R}_{2 j}$ in model (3.5) are still nonlinear, they are transformed into linear forms by variable alternation. Specifically, model (3.5) is readjusted to the following linear programming model by defining $W \cdot \hat{R}_{1 j}=\hat{r}_{1 j}$ and $W \cdot \hat{R}_{2 j}=\hat{r}_{2 j}(j=1,2, \ldots, n)$.

$$
\begin{gathered}
\max \quad U^{T} \cdot\left(\sum_{j=1}^{n} Y_{j}\right)+\Phi^{T} \cdot\left(\sum_{j=1}^{n} Z_{j}\right) \\
\text { s.t. } \quad V^{T} \cdot\left(\sum_{j=1}^{n} X_{j}\right)+\Phi^{T} \cdot\left(\sum_{j=1}^{n} Z_{j}\right)+W \cdot \hat{R}=1 ; \\
U^{T} Y_{j}-V^{T} X_{j}-\hat{r}_{1 j}-\hat{r}_{2 j} \leq 0, j=1,2, \ldots, n ; \\
\Phi^{T} Z_{j}-V^{T} X_{j}-\hat{r}_{1 j} \leq 0, j=1,2, \ldots, n ; \\
U^{T} Y_{j}-\Phi^{T} Z_{j}-\hat{r}_{2 j} \leq 0, j=1,2, \ldots, n ; \\
\sum_{j=1}^{n}\left(\hat{r}_{1 j}+\hat{r}_{2 j}\right)=W \cdot \hat{R} ; \\
\hat{r}_{1 j} \geq 0, \hat{r}_{2 j} \geq 0, j=1,2, \ldots, n ;
\end{gathered}
$$




$$
V \geq \mathbf{0}, U \geq \mathbf{0}, \Phi \geq \mathbf{0}, W>0
$$

By solving model (3.6), all supply chains will get an optimal solution $\left(V^{T *}, U^{T *}, \Phi^{T *}, W^{*}, \hat{r}_{1 j}^{*}, \hat{r}_{2 j}^{*}\right)(j=1,2, \ldots, n)$. Based on this solution, the Pareto-optimal cross-efficiency scores of all supply chains under the optimal strategy of resource allocation can be determined. Therefore, model (3.6) indicates that in order to generate a set of Pareto-optimal cross-efficiency scores, it strives to maximize the efficiency of all supply chains under the allocation of common resources. This then allows the proposed method to provide overall and better cross-efficiency scores for all supply chains. In other words, the set of cross-efficiency scores is non-dominated and does not need to be further improved. As a result, the optimal strategy of resource allocation identified through model (3.6) is more acceptable to all supply chains.

Theorem 3.2. The optimal value of the objective function for model (3.6) is always 1.

Proof. (i) First, it is clarified that the objective function of model (3.6) is not greater than 1.

The second constraint in model (3.6) can be adjusted to $U^{T} Y_{j}+\Phi^{T} Z_{j}-\left(V^{T} X_{j}+\Phi^{T} Z_{j}+\hat{r}_{1 j}+\hat{r}_{2 j}\right) \leq$ $0(j=1,2, \ldots, n)$, and we have $U^{T} Y_{j}+\Phi^{T} Z_{j} \leq V^{T} X_{j}+\Phi^{T} Z_{j}+\hat{r}_{1 j}+\hat{r}_{2 j}(j=1,2, \ldots, n)$. Furthermore, we can obtain $\sum_{j=1}^{n}\left(U^{T} Y_{j}+\Phi^{T} Z_{j}\right) \leq \sum_{j=1}^{n}\left(V^{T} X_{j}+\Phi^{T} Z_{j}+\hat{r}_{1 j}+\hat{r}_{2 j}\right)$ by summing these $n$ inequalities, and we have $U^{T} \cdot \sum_{j=1}^{n} Y_{j}+\Phi^{T} \cdot \sum_{j=1}^{n} Z_{j} \leq V^{T} \cdot \sum_{j=1}^{n} X_{j}+\Phi^{T} \cdot \sum_{j=1}^{n} Z_{j}+W \cdot \hat{R}=1$.

(ii) Next, we demonstrate that it is possible to achieve an objective function of 1 . Let $V=\left(v_{1}, v_{2}, \ldots, v_{m}\right)^{T} \in$ $R_{+}^{m}, U=\left(u_{1}, u_{2}, \ldots, u_{s}\right)^{T} \in R_{+}^{s}$ and $\Phi=\left(\varphi_{1}, \varphi_{2}, \ldots, \varphi_{D}\right)^{T} \in R_{+}^{D}$. And then, model (3.6) can be expressed as the following linear programming model.

$$
\begin{aligned}
\max & \sum_{r=1}^{s} u_{r} \cdot\left(\sum_{j=1}^{n} y_{r j}\right)+\sum_{d=1}^{D} \varphi_{d} \cdot\left(\sum_{j=1}^{n} z_{d j}\right) \\
\text { s.t. } & \sum_{i=1}^{m} v_{i} \cdot\left(\sum_{j=1}^{n} x_{i j}\right)+\sum_{d=1}^{D} \varphi_{d} \cdot\left(\sum_{j=1}^{n} z_{d j}\right)+\sum_{j=1}^{n}\left(\hat{r}_{1 j}+\hat{r}_{2 j}\right)=1 \\
& \sum_{r=1}^{s} u_{r} \cdot y_{r j}-\sum_{i=1}^{m} v_{i} \cdot x_{i j}-\hat{r}_{1 j}-\hat{r}_{2 j} \leq 0, j=1,2, \ldots, n \\
& \sum_{d=1}^{D} \varphi_{d} \cdot z_{d j}-\sum_{i=1}^{m} v_{i} \cdot x_{i j}-\hat{r}_{1 j} \leq 0, j=1,2, \ldots, n \\
& \sum_{r=1}^{s} u_{r} \cdot y_{r j}-\sum_{d=1}^{D} \varphi_{d} \cdot z_{d j}-\hat{r}_{2 j} \leq 0, j=1,2, \ldots, n \\
& \sum_{j=1}^{n}\left(\hat{r}_{1 j}+\hat{r}_{2 j}\right)=W \cdot \hat{R} \\
& \hat{r}_{1 j} \geq 0, \hat{r}_{2 j} \geq 0, j=1,2, \ldots, n \\
& v_{i} \geq 0, i=1,2, \ldots, m \\
& u_{r} \geq 0, r=1,2, \ldots, s \\
& \varphi_{d} \geq 0, d=1,2, \ldots, D \\
& W>0 .
\end{aligned}
$$


Let $z_{D}=\max _{j=1,2, \ldots, n}\left(z_{D j}\right), f=\hat{R} \cdot z_{D} \cdot \sum_{j=1}^{n} y_{s j}+\sum_{j=1}^{n} z_{D j}$, and consider $u_{s}^{\prime}=\hat{R} \cdot z_{D} / f, u_{r}^{\prime}=0(\forall r, r \neq s)$, $\varphi_{D}^{\prime}=1 / f, \varphi_{d}^{\prime}=0(\forall d, d \neq D), v_{i}^{\prime}=0(\forall i), W^{\prime}=z_{D} \cdot \sum_{j=1}^{n} y_{s j} / f, \hat{r}_{1 j}^{\prime}=z_{D j} / f, \hat{r}_{2 j}^{\prime}=\left(\hat{R} \cdot y_{s j} \cdot z_{D}-z_{D j}\right) / f$. Then $\zeta^{\prime}=\left(v^{\prime}{ }_{i}, u_{r}^{\prime}, \varphi_{d}^{\prime}, W^{\prime}, \hat{r}_{1 j}^{\prime}, \hat{r}_{2 j}^{\prime}\right)$ is a feasible solution to model (3.7) that satisfies all of the constraints in model (3.7), such that

$$
\begin{aligned}
& \sum_{i=1}^{m} v_{i} \cdot\left(\sum_{j=1}^{n} x_{i j}\right)+\sum_{d=1}^{D} \varphi_{d} \cdot\left(\sum_{j=1}^{n} z_{d j}\right)+\sum_{j=1}^{n}\left(\hat{r}_{1 j}+\hat{r}_{2 j}\right)=\sum_{j=1}^{n} z_{D j} / f+\left(z_{D} \cdot \sum_{j=1}^{n} y_{s j} / f\right) \cdot \hat{R}=1 \\
& \sum_{r=1}^{s} u_{r} \cdot y_{r j}-\sum_{i=1}^{m} v_{i} \cdot x_{i j}-\hat{r}_{1 j}-\hat{r}_{2 j}=\left(\hat{R} z_{D} / f\right) \cdot y_{s j}-z_{D j} / f-\left(\hat{R} z_{D} \cdot y_{s j}-z_{D j}\right) / f \leq 0 \\
& \sum_{d=1}^{D} \varphi_{d} \cdot z_{d j}-\sum_{i=1}^{m} v_{i} \cdot x_{i j}-\hat{r}_{1 j}=z_{D j} \cdot(1 / f)-z_{D j} / f \leq 0 \\
& \sum_{r=1}^{s} u_{r} \cdot y_{r j}-\sum_{d=1}^{D} \varphi_{d} \cdot z_{d j}-\hat{r}_{2 j}=\left(\hat{R} z_{D} / f\right) \cdot y_{s j}-z_{D j} \cdot(1 / f)-\left(\hat{R} z_{D} \cdot y_{s j}-z_{D j}\right) / f \leq 0 \\
& \sum_{j=1}^{n}\left(\hat{r}_{1 j}+\hat{r}_{2 j}\right)=\sum_{j=1}^{n}\left(z_{D j} / f+\left(\hat{R} z_{D} \cdot y_{s j}-z_{D j}\right) / f\right)=\left(\hat{R} z_{D} \cdot \sum_{j=1}^{n} y_{s j}\right) / f=W \cdot \hat{R} .
\end{aligned}
$$

And it is also easy to prove that this feasible solution satisfies $v_{i}^{\prime}, u_{r}^{\prime}, \varphi^{\prime}{ }_{d}, W^{\prime}, \hat{r}_{1 j}^{\prime}, \hat{r}_{2 j}^{\prime} \geq 0, \forall j$.

Therefore, the optimal objective function of model (3.7) with the optimal solution $\left(v_{i}^{*}, u_{r}^{*}, \varphi_{d}^{*}, W^{*}, \hat{r}_{1 j}^{*}, \hat{r}_{2 j}^{*}\right)(j=1,2, \ldots, n)$ is no less than the value of the objective function of model (3.7) with the feasible solution $\zeta^{\prime}=\left(v_{i}^{\prime}, u_{r}^{\prime}, \varphi_{d}^{\prime}, W^{\prime}, \hat{r}_{1 j}^{\prime}, \hat{r}_{2 j}^{\prime}, j=1,2, \ldots, n\right)$, as shown below.

$$
\begin{aligned}
& \sum_{r=1}^{s} u_{r}^{*} \cdot\left(\sum_{j=1}^{n} y_{r j}\right)+\sum_{d=1}^{D} \varphi_{d}^{*} \cdot\left(\sum_{j=1}^{n} z_{d j}\right) \\
& \geq \sum_{r=1}^{s} u_{r}^{\prime} \cdot\left(\sum_{j=1}^{n} y_{r j}\right)+\sum_{d=1}^{D} \varphi_{d}^{\prime} \cdot\left(\sum_{j=1}^{n} z_{d j}\right)=\left(\hat{R} z_{D} / f\right) \cdot \sum_{j=1}^{n} y_{s j}+(1 / f) \cdot \sum_{j=1}^{n} z_{D j}=1
\end{aligned}
$$

As a result, the optimal objective function of model (3.7) is 1. Furthermore, since the subscript s of the output weight is chosen randomly, there is at least one optimal solution that will allow the optimal objective function to be 1 . And it does not imply that there is only one case where the s-th output weight satisfies $u_{s}^{\prime}=\hat{R} \cdot z_{D} / f$. That is, there is at least one optimal solution such that the optimal objective function of model (3.6) is 1 .

Theorem 3.3. There is at least one optimal strategy of resource allocation, which makes DEA efficient for all supply chains as well as both enterprises within the supply chain by using model (3.6) with common weights.

Proof. Based on the optimal allocation strategy $\hat{R}_{1 j}^{\prime}=\hat{r}_{1 j}^{\prime} / W^{\prime}$ and $\hat{R}_{2 j}^{\prime}=\hat{r}_{2 j}^{\prime} / W^{\prime}$, the feasible solution $\zeta^{\prime}=\left(v_{i}^{\prime}\right.$, $\left.u_{r}^{\prime}, \varphi_{d}^{\prime}, W^{\prime}, \hat{r}_{1 j}^{\prime}, \hat{r}_{2 j}^{\prime}, j=1,2, \ldots, n\right)$ in Theorem 3.2 can be written as $\left(v^{\prime}{ }_{i}, u_{r}^{\prime}, \varphi^{\prime}{ }_{d}, W^{\prime}, \hat{R}^{\prime}{ }_{1 j}, \hat{R}^{\prime}{ }_{2 j}, j=1,2, \ldots, n\right)$. And this feasible solution allows all supply chains, as well as the upstream suppliers and the downstream manufacturers, to have Pareto-optimal cross-efficiency scores of 1, such that 


$$
\begin{gathered}
E_{j}^{(\mathrm{S})^{\prime}}=\frac{\sum_{r=1}^{s} u^{\prime}{ }_{r} y_{r j}+\sum_{d=1}^{D} \varphi^{\prime}{ }_{d} z_{d j}}{\sum_{i=1}^{m} v^{\prime}{ }_{i} x_{i j}+\sum_{d=1}^{D} \varphi^{\prime}{ }_{d} z_{d j}+W^{\prime} \cdot \hat{R}_{1 j}^{\prime}+W^{\prime} \cdot \hat{R}_{2 j}^{\prime}}=\frac{\left(\hat{R} z_{D} / f\right) \cdot y_{s j}+z_{D j} \cdot(1 / f)}{z_{D j} \cdot(1 / f)+z_{D j} / f+\left(\hat{R} z_{D} \cdot y_{s j}-z_{D j}\right) / f}=1, \forall j ; \\
E_{j}^{(1)^{\prime}}=\frac{\sum_{d=1}^{D} \varphi^{\prime}{ }_{d} z_{d j}}{\sum_{i=1}^{m} v^{\prime}{ }_{i} x_{i j}+W^{\prime} \cdot \hat{R}_{1 j}^{\prime}}=\frac{z_{D j} \cdot(1 / f)}{z_{D j} / f}=1, \forall j ; \\
E_{j}^{(2)^{\prime}}=\frac{\left(\hat{R} z_{D} / f\right) \cdot y_{s j}}{\sum_{d=1}^{D} \varphi_{r=1}^{\prime}{ }_{d} z_{d j}+W^{\prime} y_{r j} \cdot \hat{R}_{2 j}^{\prime}}=\frac{z_{D j} \cdot(1 / f)+\left(\hat{R} z_{D} \cdot y_{s j}-z_{D j}\right) / f}{z^{\prime}}=1, \forall j ; \\
\sum_{j=1}^{n}\left(\hat{R}_{1 j}^{\prime}+\hat{R}_{2 j}^{\prime}\right)=\sum_{j=1}^{n}\left[z_{D j} /\left(z_{D} \cdot \sum_{j=1}^{n} y_{s j}\right)+\left(\hat{R} z_{D} \cdot y_{s j}-z_{D j}\right) /\left(z_{D} \cdot \sum_{j=1}^{n} y_{s j}\right)\right] \\
=\sum_{j=1}^{n}\left[\left(\hat{R} z_{D} \cdot y_{s j}\right) /\left(z_{D} \cdot \sum_{j=1}^{n} y_{s j}\right)\right]=\hat{R} ;
\end{gathered}
$$

Furthermore, as described in the proof of Theorem 3.2, the subscript " $s$ " of the output weight is chosen randomly. Therefore, there is at least one optimal solution such that the Pareto-optimal cross-efficiency scores of all supply chains are 1.

\section{Application example}

\subsection{Resource allocation among sustainable supply chains}

With the rapid development of economic globalization, environmental protection is facing a huge challenge. To cope with irreversible problems such as resource shortage, environmental pollution and climate warming, sustainable development problem has gradually become a focus topic in the world. The relevant government departments strive to achieve sustainable development goals by formulating policies and regulations related to environmental protection, developing the awareness of environmental protection, and promoting the idea of green development. Therefore, the relevant departments provide materials or funds to encourage enterprises with sustainable development ability to engage in sustainable operation activities. These financial subsidies can not only appropriately alleviate the financial shortage faced by enterprises in insisting on sustainable operation, but also promote more enterprises to adopt the idea of green development and to drive the sustainable development of the global economy. Therefore, this paper explores how to improve the operational efficiency of sustainable supply chains for 27 Iranian resin production companies, and develops an optimal strategy of resource allocation to ensure the efficient operation of sustainable supply chains.

There are 27 sustainable supply chains, each of which is a two-stage structure system consisting of an upstream supplier and a downstream manufacturer. Therein, the upstream supplier uses production costs $x_{1}$, employee annual turnover rates $x_{2}$, and environmental $\operatorname{costs} x_{3}$ to obtain products $z_{1}$ and to provide cooperative costs $z_{2}$ for the green production plan. It is noted that $x_{3}$ invested in the upstream supplier is the cost of green efforts to deal with environmental pollution in the production process, which is completely different from cooperative costs $z_{2}$ provided by the upstream supplier to the downstream manufacturer for the joint scheme of green production. And then, for the downstream manufacturer, the intermediate products $z_{1}$ and $z_{2}$ provided by the upstream supplier are used as inputs to produce trained personnel $y_{1}$, green products $y_{2}$ and revenue $y_{3}$. And the relevant data is derived from [12]. In addition, with the existing technology and operation scale of the supply chain, it is 
assumed that the relevant government departments will provide $100,000\left(10^{3} \$\right)$ in financial to encourage supply chains with environmental protection technologies to implement sustainable development schemes.

First of all, model (3.6) is used to evaluate the operational efficiency of 27 sustainable supply chains without involving common resource allocation. As a result, the parameters with respect to common resources in model (3.6) are set to zero, and the Pareto-optimal cross-efficiency scores of 27 sustainable supply chains and those of two enterprises within the supply chain are determined by applying this model, as shown in the second to fourth columns of Table 1. From the optimal results of the performance evaluation, it can be found that (1) the Pareto-optimal cross-efficiency scores of 27 sustainable supply chains before implementing the allocation strategy of common resource are less than 1, which indicates that all supply chains fail to achieve DEA efficient. (2) The two-stage sustainable supply chain achieves DEA efficient if and only if the efficiency scores of both the upstream supplier and the downstream manufacturer within this supply chain are 1. Even if there is an efficient enterprise, the operational efficiency of the whole supply chain still fails to achieve DEA efficient because the other enterprise is still inefficient.

The next study explores the performance evaluation of 27 sustainable supply chains after the resource allocation. Therefore, assuming that the existing common resource is $\hat{R}=100000\left(10^{3} \$\right)$, model (3.6) is applied to measure the Pareto-optimal cross-efficiency scores of 27 two-stage supply chains and those of two enterprises within the supply chain after the implementation of resource allocation, as shown in the fifth to seventh columns of Table 1. And the corresponding optimal allocation results of common resource are shown in the eighth to tenth columns of Table 1.

From the evaluation results, it can be found that the Pareto-optimal cross-efficiency scores of all two-stage supply chains after the allocation of common resources are 1 . This indicates that the financial subsidies, provided by government departments to support the green development of supply chains, have a positive effect on improving the operational efficiency of supply chains under reasonable resource allocation, which is mainly reflected in two aspects. On the one hand, since sustainable supply chains require additional investment in green development technologies, environmental protection costs and pollution control costs, financial subsidies provided by the relevant government departments can appropriately alleviate the financial difficulties faced by supply chains in striving to green development. Therefore, the rational allocation of financial subsidies will play a significant role in improving the operation performance of sustainable supply chains. On the other hand, financial subsidies are limited and cannot meet the financial demands of all sustainable supply chains. Therefore, the reasonable allocation strategy can maximize the utility of financial resources. As a result, this paper uses a two-stage cross-efficiency model based on Pareto optimality to evaluate the operational efficiency scores of sustainable supply chains and to explore the optimal allocation strategy of common resources. The model not only achieves optimal allocation of common resources, but also ensures that all sustainable supply chains as well as both the upstream supplier and the downstream manufacturer within the supply chain achieve DEA efficient in the resource allocation process. Therefore, this optimal allocation strategy is more easily accepted by all sustainable supply chains, which promotes the implementation of this strategy, so as to achieve a win-win result in terms of optimal resource allocation and efficient business operation.

\subsection{Management implications of the proposed model}

The common resources provided by the relevant government departments are always relatively scarce compared to the demand of sustainable supply chains, and how to reasonably allocate the limited resources and to improve the efficiency scores of all supply chains are the concerns of both the relevant government departments and all supply chains. Specifically, from the perspective of the relevant government departments, a given quantity of common resources is provided to encourage all sustainable supply chains to optimize their operational processes and to improve the sustainability of the relevant industrial sectors. In other words, the relevant government departments regard these sustainable supply chains as a whole unit and strive to maximize the overall efficiency of this unit. From the perspective of sustainable supply chains, each sustainable supply chain strives to maximize its cross-efficiency. And in order to ensure that the cross-efficiency of sustainable supply chains is not worse during the cross-evaluation process, each self-interested supply chain must guarantee that the 


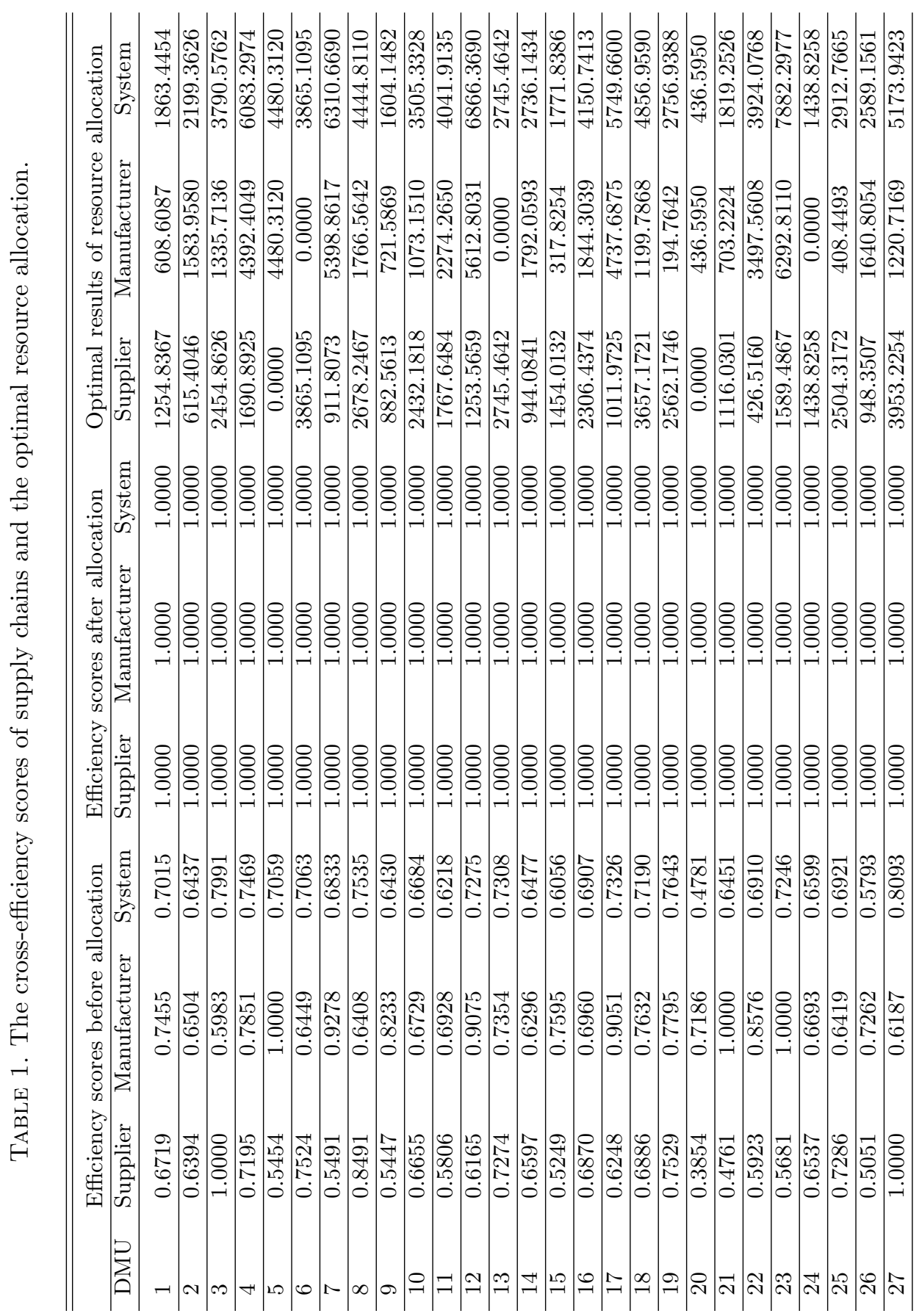


self-evaluated efficiency is at least not smaller than its peer-evaluated efficiencies generated from the other supply chains. Therefore, the proposed model tries to maximize the cross-efficiency scores of all sustainable supply chains, and allows all supply chains to choose a performance evaluation mechanism and an optimal allocation of common resources together, so that all supply chains that obtain the optimal amount of common resources can obtain overall and better Pareto-optimal cross-efficiency scores through this evaluation mechanism. Moreover, the results of practical application show that 27 Iranian resin production companies have made full use of the common resources provided by government departments, and all of them have achieved DEA efficient after the implementation of the optimal allocation strategy. This is consistent with the motivation of government departments that want to promote sustainable supply chains to optimize their operational efficiencies and to improve the sustainable operation of the relevant industrial sectors. Moreover, the proposed model maximizes the weighted average of the cross-efficiency scores of the 27 sustainable supply chains, not the cross-efficiency score of every single supply chain. This is consistent with the motivation of all supply chains striving to maximize their own interests.

\section{Conclusion}

This paper focuses on the allocation strategy of common resources among two-stage structure supply chains. With the rapid development of social economy, the contradiction between the scarcity of common resources and the ever-growing demands of social entities for these resources is also becoming increasingly obvious. Therefore, it is especially important to develop a reasonable and efficient strategy of resource allocation to achieve the optimal allocation of common resources. In addition, most of the production or service systems in the real-world environment are composed of multiple subdivisions coordinating with each other to cope with the pressure of market competition. As a result, it is clear that exploring the internal processes of an organization system contributes to the analysis of the factors that influence organizational development. Since efficiency is one of the most important criteria to measure the level of organizational development, this paper objectively evaluates the operational efficiency of production or service systems by using a non-parametric DEA method. To further improve the discrimination power of DEA models and achieve the Pareto optimality of the efficiency evaluation results, this paper takes the self-interested behavior of enterprises as the Pareto improvement principle in the cross-evaluated situation. Taking the two-stage supply chain in which the upstream supplier provides intermediate products to the downstream manufacturer for production as the research object, this paper proposes a two-stage cross-efficiency model based on Pareto optimality to determine the Pareto-optimal weights profile, and the Pareto-optimal cross-efficiency scores of all supply chains after the resource allocation are calculated. The following three conclusions can be drawn from the proposed model.

First of all, in the situation involving the allocation of common resources, the cross-efficiency of a two-stage supply chain can be decomposed into the weighted sum of the cross-efficiency of the upstream supplier and the cross-efficiency of the downstream manufacturer, where the stage weight of each enterprise is the cross-weight obtained by the arithmetic average of the self-evaluated weight and the peer-evaluated weight. Secondly, the proposed model allows all supply chains to choose a common set of weights that result in Pareto-optimal crossefficiency scores for all supply chains as well as the upstream suppliers and the downstream manufacturers. Thirdly, the proposed model not only identifies an optimal allocation strategy of common resources, but also enables all supply chains as well as the upstream supplier and the downstream manufacturer within the supply chain to achieve DEA efficient after implementing that strategy.

Finally, the effectiveness of the proposed model is illustrated by applying the practical application with respect to the sustainable supply chains of 27 Iranian resin production companies. In addition, it is assumed that the relevant government departments provide financial subsidies to encourage supply chains to implement green production and sustainable operation, but these subsidies cannot fully meet the financial demands of all supply chains. Therefore, it is necessary to provide an optimal resource allocation strategy. As a result, the Pareto-optimal two-stage cross-efficiency model proposed in this paper to solve the resource allocation problem can achieve a win-win result. Specifically, compared to the Pareto-optimal cross-efficiency results of 
supply chains without considering resource allocation, the proposed model can identify an optimal allocation strategy of common resources to make all sustainable supply chains DEA efficient. Moreover, the same goal of all sustainable supply chains is to allow them to have an efficiency score of 1 . Therefore, this Pareto-optimal allocation result of common resources is more easily adopted by all sustainable supply chains, which facilitates the implementation of that strategy.

In future research, we can extend the proposed model to the case of variable returns to scale. And note that this paper explores two-stage supply chains with the most basic structure, but it does not consider generalized two-stage structure systems. For example, only partial outputs from the upstream supplier become the inputs in the downstream manufacturer and the downstream manufacturer has other external inputs in addition to those produced by the upstream supplier. In addition, most of the actual supply chains may have multiple stages, and the problem of resource allocation among multi-stage supply chains is quite common in reality. Therefore, we can further extend the proposed model to the resource allocation among generalized two-stage systems or the resource allocation among multi-stage systems in future research.

Acknowledgements. The authors are grateful to the Editor-in-chief, Associate editors and anonymous reviewers for their constructive comments and suggestions. This work is supported by the National Natural Science Foundation of China under grant (No. 71671095), the Tianjin Philosophy and Social Science Planning Key Project (TJGL21-009).

\section{REFERENCES}

[1] Q. An, P. Wang, A. Emrouznejad and J. Hu, Fixed cost allocation based on the principle of efficiency invariance in two-stage systems. Eur. J. Oper. Res. 283 (2020) 662-675.

[2] A. Charnes and W.W. Cooper, Programming with linear fractional functionals. Nav. Res. Logist. Q. 9 (1962) $181-186$.

[3] A. Charnes, W.W. Cooper and E. Rhodes, Measuring the efficiency of decision making units. Eur. J. Oper. Res. 2 (1978) 429-444.

[4] Y. Chen, W.D. Cook, N. Li and J. Zhu, Additive efficiency decomposition in two-stage DEA. Eur. J. Oper. Res. 196 (2009) 1170-1176.

[5] L. Chen, Y. Huang, M.J. Li and Y.M. Wang, Meta-frontier analysis using cross-efficiency method for performance evaluation. Eur. J. Oper. Res. 280 (2020) 219-229.

[6] M. Chen, S. Ang, L. Jiang and F. Yang, Centralized resource allocation based on cross-evaluation considering organizational objective and individual preferences. OR Spectr. 42 (2020) 529-565.

[7] W.D. Cook and J. Zhu, DEA Cobb-Douglas frontier and cross-efficiency. J. Oper. Res. Soc. 65 (2014) $265-268$.

[8] M. Davtalab-Olyaie and M. Asgharian, On Pareto-optimality in the cross-efficiency evaluation. Eur. J. Oper. Res. 288 (2021) $247-257$.

[9] J. Doyle and R. Green, Efficiency and cross-efficiency in DEA: Derivations, meanings and uses. J. Oper. Res. Soc. 45 (1994) $567-578$.

[10] J. Du, W.D. Cook, L. Liang and J. Zhu, Fixed cost and resource allocation based on DEA cross-efficiency. Eur. J. Oper. Res. 235 (2014) 206-214.

[11] C. Kao and S.T. Liu, Cross efficiency measurement and decomposition in two basic network systems. Omega 83 (2019) 70-79.

[12] M. Khodakarami, A. Shabani, R.F. Saen and M. Azadi, Developing distinctive two-stage data envelopment analysis models: An application in evaluating the sustainability of supply chain management. Measurement 70 (2015) 62-74.

[13] F. Li, Q. Zhu and L. Liang, Allocating a fixed cost based on a DEA-game cross efficiency approach. Expert. Syst. Appl. 96 (2018) 196-207.

[14] F. Li, Y. Wang, A. Emrouznejad, Q. Zhu and G. Kou, Allocating a fixed cost across decision-making units with undesirable outputs: A bargaining game approach. To appear in: J. Oper. Res. Soc. (2021). DOI: 10.1080/01605682.2021.1981781.

[15] L. Liang, W.D. Cook and J. Zhu, DEA models for two-stage processes: Game approach and efficiency decomposition. Nav. Res. Logist. (NRL) $\mathbf{5 5}$ (2008) 643-653.

[16] L. Liang, J. Wu, W.D. Cook and J. Zhu, The DEA game cross-efficiency model and its Nash equilibrium. Oper. Res. 56 (2008) $1278-1288$.

[17] S. Lim, Minimax and maximin formulations of cross-efficiency in DEA. Comput. Ind. Eng. 62 (2012) $726-731$.

[18] C.Q. Ma, D.B. Liu, Z.B. Zhou, W. Zhao and W.B. Liu, Game cross efficiency for systems with two-stage structures. J. Appl. Math. (2014) 747596.

[19] F.Y. Meng and B.B. Xiong, Logical efficiency decomposition for general two-stage systems in view of cross efficiency. Eur. J. Oper. Res. 294 (2021) 622-632.

[20] H.H. Örkcü, V.S. Özsoy, M. Örkc and H. Bal, A neutral cross efficiency approach for basic two stage production systems. Expert. Syst. Appl. 125 (2019) 333-344. 
[21] S.J. Sharahi and K. Khalili-Damghani, Fuzzy type-II De-Novo programming for resource allocation and target setting in network data envelopment analysis: A natural gas supply chain. Expert. Syst. Appl. 117 (2019) 312-329.

[22] T.R. Sexton, R.H. Silkman and A.J. Hogan, Data envelopment analysis: Critique and extensions. New Dir. Progr. Eval. 1986 (1986) $73-105$.

[23] X. Shao and M.Q. Wang, Two-stage cross-efficiency evaluation based on prospect theory. To appear in: J. Oper. Res. Soc. (2021). DOI: 10.1080/01605682.2021.1918587.

[24] H. Sharafi, F.H. Lotfi, G.R. Jahanshahloo and S. Razipour-GhalehJough, Fair allocation fixed cost using cross-efficiency based on Pareto concept. Asia. Pac. J. Oper. Res. 37 (2020) 1950036.

[25] Y.M. Wang and K.S. Chin, A neutral DEA model for cross-efficiency evaluation and its extension. Expert. Syst. Appl. 37 (2010) 3666-3675.

[26] Y.M. Wang, K.S. Chin and P. Jiang, Weight determination in the cross-efficiency evaluation. Comput. Ind. Eng. 61 (2011) 497-502.

[27] J. Wu, W.Y. Zhang, S. Zhang, Y.N. Liu and X.H. Meng, A matrix-based Bayesian approach for manufacturing resource allocation planning in supply chain management. Int. J. Prod. Res. 51 (2013) 1451-1463.

[28] J. Wu, J.F. Chu, J.S. Sun and Q.Y. Zhu, DEA cross-efficiency evaluation based on Pareto improvement. Eur. J. Oper. Res. 248 (2016) 571-579.

[29] J. Wu, J.F. Chu, J.S. Sun, Q.Y. Zhu and L. Liang, Extended secondary goal models for weights selection in DEA cross-efficiency evaluation. Comput. Ind. Eng. 93 (2016) 143-151.

[30] Q.W. Xie, R. Li, Y.P. Zou, Y.J. Liu and X.J. Wang, A two-stage method for improving discrimination and variable selection in DEA models. To appear in: IMA J. Manag. Math. (2021) dpab023. DOI: 10.1093/imaman/dpab023.

[31] M.M. Yu, L.H. Chen and B. Hsiao, A fixed cost allocation based on the two-stage network data envelopment approach. J. Bus. Res. 69 (2016) 1817-1822.

[32] J.Y. Zhao, X. Xi, S.S. Wang and C.Y. Gong, Dynamic analysis of different resource allocations: Implications for resource orchestration management of strategic alliances. Comput. Ind. Eng. 158 (2021) 107393.

[33] Q.Y. Zhu, J. Wu, X.C. Li and B.B. Xiong, China's regional natural resource allocation and utilization: A DEA-based approach in a big data environment. J. Clean. Prod. 142 (2017) 809-818.

\section{Subscribe to Open (S20)}

\section{A fair and sustainable open access model}

This journal is currently published in open access under a Subscribe-to-Open model (S2O). S2O is a transformative model that aims to move subscription journals to open access. Open access is the free, immediate, online availability of research articles combined with the rights to use these articles fully in the digital environment. We are thankful to our subscribers and sponsors for making it possible to publish this journal in open access, free of charge for authors.

\section{Please help to maintain this journal in open access!}

Check that your library subscribes to the journal, or make a personal donation to the $\mathrm{S} 2 \mathrm{O}$ programme, by contacting subscribers@edpsciences.org

More information, including a list of sponsors and a financial transparency report, available at: https://www. edpsciences.org/en/maths-s2o-programme 